Estudios sobre armas antiguas, armamento, arte militar $\mathrm{y}$ vida cultural en oriente y occidente

XXVI (2006), pp. 105-117

ISSN: 0435-029X

\title{
UNA CUESTIÓN A DEBATE: LA LORICA SEGMENTATA EN LAS FRONTERAS ORIENTALES DEL IMPERIO ROMANO
}

POR

\author{
A. Raúl MenÉndeZ Argüín*
}

\section{RESUMEN - ABSTRACT}

En el presente artículo se exponen, tras una profunda revisión de las evidencias y fuentes disponibles, toda una serie de argumentos que ponen de manifiesto la inadecuación de la coraza de placas articuladas de época romana, más conocida como lorica segmentata, a los teatros de operaciones de las fronteras orientales. A estos argumentos se une la ausencia casi total (con una sola excepción) de hallazgos de segmentata en estas provincias del Este mediterráneo, lo que, si bien no es un argumento decisivo, supone un hándicap importante de aquellas teorías que defienden un empleo masivo de este tipo de coraza a lo largo y ancho de todo el Imperio.

This paper, after a deep revision of the evidences and available sources, proposes a series of arguments that show the inadequacy of Roman plate armour, better known as lorica segmentata, to the theaters of operations of the oriental border of the Empire. To these arguments it can be added the almost total absence (with a single exception) of segmentata finds in these provinces of the Roman East. Although it is not a decisive argument, these absences suppose an important drawback to those theories that defend a massive employment of this armour type along the whole Empire.

\section{PALABRAS CLAVE - KeYWORDS}

Armas defensivas. Corazas. Ejército romano. Legión romana. Legionario. Oriente romano.

Defensive weapons. Armour. Roman Army. Roman Legion. Legionary infantryman. Roman East.

\section{INTRODUCCIÓN}

La coraza de placas ${ }^{1}$, cuyo origen hoy día sabemos que hay que situar a fines del principado de Augusto 2 , se convirtió a lo largo del s. I d.C. y durante todo el s. II en la protección

* Becario Postdoctoral, Universidad Paris Sorbonne-Paris IV. Este trabajo ha sido desarrollado gracias a la Beca (EX2003-0645) del programa de Becas Postdoctorales en España y en el Extranjero del Ministerio de Educación, Cultura y Deporte, desarrollada en la École Doctorale «Mondes Anciens et Medievaux» (ED 0022) de la Université Paris Sorbonne-Paris IV, entre noviembre de 2003 y noviembre de 2005. El presente artículo también se enmarca en el Grupo de Investigación «La Bética Romana: su Patrimonio Histórico» (Grupo n. ${ }^{\circ}$ HUM-0323 del II Plan Andaluz de Investigación); Departamento de Historia Antigua, Universidad de Sevilla (1997-2004). Investigador Principal: Dr. Genaro Chic García.

Quiero agradecer asimismo la lectura del manuscrito a los Doctores G. Chic García, A. Caballos Rufino y Yann Le Bohec, cuyas acertadas apreciaciones han contribuido sin duda a mejorar el resultado final de este trabajo. Huelga decir que cualquier posible error o imprecisión es única y exclusivamente achacable al autor.

1 El término lorica segmentata es una denominación moderna, empleada a partir del Renacimiento, pero 
tipo de los legionarios romanos. Con ella aparecen representados en los grandes relieves históricos (Columna Trajana ${ }^{3}$, fragmentos del Gran Friso de Trajano ${ }^{4}$, base de la Columna Antonina $^{5}$, Columna Aureliana ${ }^{6}$, Arco de Septimio Severo ${ }^{7}$, relieves de época de Marco Aurelio incorporados en el Arco de Constantino), pero hasta qué punto era cierta esta estandarización y, sobre todo, en qué zonas de las fronteras imperiales era aquélla la imagen propia del legionario es un tema sujeto en la actualidad a discusión.

Antes de plantear los términos del debate creemos necesario efectuar una exposición relativamente detallada de la pieza de equipo defensivo que nos ocupa. La lorica segmentata es un tipo de coraza formada por placas de metal rígido, unidas entre sí mediante tiras de cuero interiores para mantenerlas en posición, que protegía el tronco del soldado desde la cintura hasta el cuello, con piezas adaptadas también para proteger los hombros. Este tipo de coraza es la que tradicionalmente se ha asociado con las tropas legionarias; pero, si bien es cierto que su empleo quedaba restringido a las legiones, sin embargo coexistió en estas unidades tanto con la coraza de escamas como con la cota de mallas ${ }^{8}$.

Hasta las recientes excavaciones en la zona de Kalkriese, donde se ha identificado de forma definitiva el desastre de Varo de 9 d.C. en el que fueron aniquiladas las legiones XVII, XVIII y XIX, el origen de este tipo de coraza se remontaba como muy pronto al segundo tercio del s. I d.C., aproximadamente en los últimos años del reinado de Tiberio9. Se quiso conectar el desarrollo de esta coraza articulada con el equipo gladiatorio, aduciéndose un pasaje de Tácito respecto a los gladiadores de la ciudad de Autún, reclutados durante la revuelta de Floro y Sacrovir para enfrentarse a las legiones ${ }^{10}$. Éstos sólo pudieron ser derribados cuando los legionarios emplearon sus hachas y zapapicos (Tácito, Annales 3.46). Los soldados y sus mandos habrían quedado tan impresionados por las cualidades defensivas de este tipo de armaduras como para comenzar su adaptación a las unidades

gracias a su gran aceptación es el que utilizaremos a lo largo de todo el artículo. Contra Simkins, que propone la denominación de lorica laminata, pues etimológicamente el término segmentata habría tenido poco que ver con las protecciones corporales (vid. Simkins, 1990a: 11).

2 Gracias a los hallazagos de Kalkriese, ver infra.

${ }^{3}$ El gran trabajo de edición de la columna fue acometido a fines del s. XIX por Cichorius, cuya obra sigue siendo de obligada referencia.

${ }^{4}$ Leander Touati, 1987.

5 La decoración escultórica se limitaba exclusivamente a la base (cf. F. Coarelli, 1980: 303-4; R. Turcan, 1995: 192; M. Tarpin, 2001: 287).

6 Petersen, Von Domaszewski y Calderini, 1896; Zwikker, 1941.

7 Brilliant, 1967.

8 Como puede observarse claramente en el monumento de Adamklissi, erigido para conmemorar las victorias dacias de Trajano. Cf. Florescu, 1965; Bianchi, 1988; Florescu, 1998.

9 Robinson (1974: 5-12) incluso retrasaba aún más esa fecha hasta hacerla coincidir aproximadamente con la invasión de Britania por Claudio. Para Harmand (1987: 197) la inclusión de la lorica segmentata en el equipamiento legionario habría que situarla a comienzos de época flavia, afirmando que las pruebas aducidas para una datación más temprana no eran concluyentes. Simkins (1986: 15), por contra, adelantaba su fecha de aparición, conectándola con la necesidad de equipar nuevas unidades creadas para compensar el desastre de Varo en Germania en 9 d.C.; a esta teoría se oponía Peterson (1996: 16), quien recordaba que sólo unas décadas antes Augusto había licenciado unas 30 legiones -bien es cierto que de efectivos reducidos-, lo cual significaría que decenas de miles de cotas de malla se encontrarían almacenadas en las distintas armerías imperiales a lo largo del mundo romano; no obstante, la posibilidad de que fueran refundidas para realizar nuevas protecciones corporales o piezas de equipo tampoco puede desecharse. Todo este debate ha recibido un nuevo vuelco tras los hallazgos de restos de segmentata de Kalkriese y otros campamentos augusteos de Germania, lo que nos obliga a adelantar el nacimiento de esta coraza como muy tarde a la segunda década antes de nuestra era (ver infra).

10 «Se añadieron los esclavos destinados al oficio de gladiadores, que, según una costumbre nacional, llevan una armadura completa de hierro; los llaman crupelarios, y son incapaces de causar heridas, pero impenetrables a la hora de recibirlas» (Tac., Annales 3.43). Han aparecido restos de segmentata en el campamento de Aulnay-deSaintonge, cuya ocupación se fecha entre 20 y 30 d.C. y que fue establecido justo tras la supresión de esta revuelta (cf. Feugère, 1993: 132). 
legionarias ${ }^{11}$. No obstante, las excavaciones en Kalkriese, donde se han identificado de forma clara restos de lorica segmentata temprana, obligan a adelantar la fecha de incorporación de esta coraza al equipo legionario al menos en medio siglo, hasta la última etapa del reinado de Augusto ${ }^{12}$. De hecho, ya se habla de un nuevo tipo de segmentata, el «tipo Kalkriese», del que debido a los escasos restos disponibles aún no existe una reconstrucción definitiva medianamente fiable ${ }^{13}$. Esta pieza de equipo defensivo habría que conectarla, por tanto, con las reformas militares del último período del gobierno de este emperador, que dispuso la fabricación de nuevos modelos de armas, tanto ofensivas como defensivas ${ }^{14} \mathrm{con}$ el fin de mejorar la protección y la eficacia de sus tropas ${ }^{15}$.

Dos fueron los modelos de lorica segmentata que se desarrollaron a lo largo de los siglos I y II, el denominado «Corbridge», en sus tres subtipos «A» $\mathrm{y}$ «B/C» ${ }^{16}$ (este último sería una variante menor del subtipo «B»), caracterizado por sus numerosos enganches, hebillas y apliques metálicos (hasta un total de 50), que constituían el elemento más débil de la armadura; y el tipo «Newstead», más funcional y con menos enganches susceptibles de romperse (concretamente 24), desarrollado a partir de comienzos del s. II ${ }^{17}$. Este modelo de coraza, en la que parece que se consigue un buen equilibrio entre protección, operatividad y costes de producción y mantenimiento, es el que se mantuvo en uso en las legiones hasta más allá de mediados del s. III d.C.

Durante la segunda mitad del s. II en las representaciones escultóricas aparece un tipo de segmentata cuyas placas se extienden hasta llegar al cuello, obviando las piezas de pectorales y dorsales propias de los tipos Kalkriese, Corbridge y Newstead. Se ha pensado en un

11 Por otra parte, no habría sido la primera vez que las prácticas gladiatorias hubieran influido en el ejército; sólo hemos de recordar, por ejemplo, el entrenamiento que el cónsul P. Rutilio Rufo dio a sus soldados a fines del s. II a.C. para enfrentarse a cimbrios y teutones (Val. Max. 2.3.2), aunque finalmente estas legiones entrenadas según los usos gladiatorios fueron conducidas por Mario en el campo de batalla. Véase también Coulston, 1998.

12 Schlüter et alii, 1992; Wilson y Creighton, 1999; Schlüter, 1999: 136 y figura 5.6; las bases augústeas de Haltern y Dangstetten en Germania también han proporcionado algunos restos de segmentata temprana, que habría que fechar (a partir del material cerámico asociado) hacia 16-15 a.C. (Trier, 1989: Abb. 105; Fingerlin, 1986: lám.7, n. ${ }^{\circ}$ 285.5, 332.2 y 544.13; Roth-Rubi, 2001; Cowan, 2004: 31-32).

13 Bishop, 2002: 23-9. Este autor ha identificado incluso dos subtipos, «A» $\mathrm{y} « \mathrm{~B} »$, si bien la reconstrucción de esta coraza propuesta en la pg. 28 es altamente hipotética. El tipo Kalkriese B se ha identificado también en Chichester y Waddon Hill, por lo que se mantuvo en uso al menos hasta 43 d.C. (véase nota 16).

14 Recordemos, por ejemplo, la introducción del scutum rectangular.

15 Hay que tener en cuenta, sin embargo, que los ejércitos a lo largo de toda la Historia se han caracterizado por una cierta continuidad en el empleo del armamento y material; debido a los costes de fabricación las armas se intentan mantener en uso el mayor tiempo posible. Por otra parte, las innovaciones armamentísticas no se introducen en ningún ejército de una sola vez, sino mediante un proceso gradual de sustitución del armamento antiguo por el más moderno, lo que hace coexistir durante un período de tiempo más o menos amplio los diferentes tipos. Tampoco hay que olvidar que los avances en cuestiones de armamento y equipo no siempre están relacionados con órdenes derivadas del mando supremo, sino que las propias unidades pueden desarrollar sobre el terreno (como sin duda ocurrió en el ejército romano) avances que otorguen ciertas ventajas a sus integrantes sobre los enemigos a los que tienen que hacer frente; en este sentido podemos evocar las manicae protectoras del brazo derecho que los legionarios emplearon en las guerras dacias del reinado de Trajano, según se desprende del monumento de Adamklissi.

16 Bishop, 2002: 31-45. El tipo Corbridge sería una evolución del tipo Kalkriese «B», que sería mejorado en toda una serie de aspectos técnicos.

17 Robinson, 1975: 180-4. Los hallazgos de segmentata de Newstead han sido de nuevo analizados por M.C. Bishop, poniéndolos en relación con otros procedentes tanto de Gran Bretaña como del continente y que también pertenecerían a este nuevo tipo de coraza de placas; a partir de todas estas evidencias el mencionado autor ha elaborado una nueva reconstrucción, que modifica en ciertos aspectos las propuestas por Robinson en 1975 (Cf. Bishop, 1999; Bishop, 2002: 46-61; la conclusión más importante a la que llega este investigador es que si bien nos encontramos ante un nuevo tipo de segmentata, las modificaciones no fueron tan revolucionarias como en un principio pudiera parecer, manteniendo numerosas características en común con el tipo Corbridge e incluso con el tipo inicial de Kalkriese). 
nuevo modelo de coraza, si bien, según nuestra opinión, sólo sería una forma estilizada del tipo Newstead en la que el artista se ha tomado la licencia de continuar con las placas hasta el cuello, sin tener en cuenta el diseño real de ese modelo de lorica. Por otra parte, no han aparecido fragmentos en el registro arqueológico que sustenten este supuesto nuevo tipo de segmentata ${ }^{18}$.

Tradicionalmente se había pensado que la lorica segmentata desapareció del equipo legionario durante la segunda mitad del s. II (la última representación escultórica de la segmentata la observamos en el Arco de Septimio Severo y los fragmentos de coraza de este tipo más tardíos databan de época antonina). Sin embargo, descubrimientos recientes de restos de segmentata en contextos fechados a lo largo del s. III, sobre todo en Britania ${ }^{19}$ y el limes germano $^{20}$, indicarían la continuidad de su empleo por lo menos hasta la década de $260^{21}$.

Por lo que concierne a sus cualidades defensivas, éstas son superiores en algunos aspectos a las de la malla, al permitir detener golpes más contundentes sin sufrir daños. Otorgaba también una buena protección ante flechas, dardos y jabalinas ${ }^{22}$; pero cuando la segmentata era especialmente efectiva era contra los golpes descendentes de espada, pues poseía unas protecciones para los hombros bastante fuertes. Además, el combatiente conservaba una amplia libertad de movimientos gracias a las articulaciones de las placas entre sí. Otras ventajas respecto a la malla son su inferior coste de fabricación y su menor peso, que podía oscilar entre los seis y los nueve kilos, dependiendo del grosor de las placas. No obstante, también contaba con serios inconvenientes. En primer lugar, el mantenimiento debía realizarse en talleres más o menos especializados y la debilidad de los enganches y hebillas (realizados en aleación de cobre ${ }^{23}$ ) obligaría a continuas rondas de reparaciones ${ }^{24}$. Este último problema se solucionó en parte con el modelo «Newstead», que, a pesar de su funcionalidad, también exigía un mantenimiento considerable ${ }^{25}$. Otro inconveniente de la segmentata respecto a la malla era la pérdida de protección para los muslos y parte superior de los brazos ${ }^{26}$. Finalmente, hay

18 Bishop, 2002: 9-12.

19 Caruana, 1993.

20 Cf. Bishop, 2002: 91; Menéndez Argüín, 2004: 214. Por otra parte, J. Aurrecoechea ha confirmado en el II Congreso de Arqueología Militar Romana, celebrado en la Universidad de León en octubre de 2004, la existencia de un taller de la VII Gemina en actividad a fines del s. III y en el que se han hallado restos de apliques metálicos en cobre/bronce destinados a la fabricación o mantenimiento de loricae segmentatae.

21 Véase Coulston, 1990: 147; Bishop, 2002: 46. Una escultura procedente del campamento legionario de Alba Iulia, Rumanía, fechada a fines del s. II o primera mitad del s. III, resulta bastante interesante porque presenta una lorica segmentata pero con los hombros protegidos por piezas de lorica squamata; en el brazo derecho el soldado porta una manica lamminata (Coulston, 1995). Bishop (2002: 62-5) incluso considera esta coraza como un nuevo tipo de segmentata, si bien al ser ésta la única evidencia no pueden extraerse conclusiones definitivas.

22 Era sin embargo vulnerable a los proyectiles lanzados desde máquinas de guerra que, según reconstrucciones recientes lograban atravesar sus placas y hundirse profundamente en el cuerpo del portador. Otra característica interesante relativa a este tipo de ataques de scorpio y cheiroballistra era la onda de choque provocada por el proyectil, que habría provocado graves roturas en los órganos internos, incapacitando o acabando directamente con la víctima (así, la onda de choque es un elemento muy importante a tener en cuenta, pues recuerda que no es necesaria la penetración física de un proyectil a través de la armadura para incapacitar o matar) (Wilkins, 2000: especialmente 92-4).

23 Concretamente orichalcum, una aleación al 80-85\% de cobre y 20-15\% de zinc (Bishop, 2002: 77).

24 Además, las reacciones químicas entre los enganches de aleación de cobre y las placas de hierro favorecían la corrosión, provocando que muchos de estos enganches se desprendieran demasiado fácilmente. De este modo, cabe la posibilidad de que la gran cantidad de restos de segmentata hallados pueda tergiversar la imagen de su expansión real, pues estos apliques tenían grandes probabilidades de entrar en el registro arqueológico. Por otra parte, en ningún momento se observa (a partir de las fuentes disponibles) el abandono por parte de los legionarios de los otros dos tipos de coraza conocidos (mallas y escamas).

25 Bishop, 2002: 84-86.

26 Cuando era necesario, no obstante, se protegía también el brazo derecho mediante placas, denominadas manicae, como puede observarse en los relieves del Tropaeum Traiani de Adamklissi (Rumania). En éstos aparecen legionarios con el brazo derecho protegido y el izquierdo desnudo, pues éste último ya contaba con la protec- 
que mencionar que esta coraza era compleja de endosar, pues el soldado necesitaba ayuda para evitar estropear las correas de sujeción, hebillas y bisagras que, como hemos visto, eran las partes más débiles de la lorica.

Buena parte de la investigación sostiene que el empleo de este modelo de armadura estaba esencialmente restringido a las legiones de las fronteras europeas y, dentro de ellas, especialmente a las tropas de Britania y ambas Germanias, donde (junto a la Dacia) ${ }^{27}$ se han producido casi todos los hallazgos de restos de este tipo de coraza ${ }^{28}$.

\section{LA SEGMENTATA EN LAS FRONTERAS ORIENTALES}

El Profesor G. Brizzi ha planteado, por su parte, una visión completamente opuesta a la teoría tradicional mencionada más arriba, considerando que el empleo de este tipo de coraza

ción proporcionada por el escudo. Otras evidencias iconográficas de su empleo entre las tropas legionarias son dos tumbas de legionarios de Mainz fechadas a mediados del s. I d.C. Restos de este tipo de protección han sido identificados en Carnuntum, Trimontium (Newstead), Corbridge, Richborough, Eining, Carlisle, Colonia Ulpia Traiana Sarmizegethusa (Rumanía) y León. Según M. Simkins, a diferencia de las manicae de tipo gladiatorio, las manicae militares sólo encierran el brazo del portador en sus dos tercios; la explicación para esta importante diferencia puede atribuirse al hecho de que encerrarlo por completo producía cierta restricción de movimientos en el codo (esta falta de movimiento del brazo era inaceptable para el ejército, aunque pudiera haber sido pasable en la arena); M.C. Bishop, ha propuesto por su parte que la manica no cubría la parte trasera del brazo, sino la delantera, al ser la más expuesta según se desprende de la técnica de combate del legionario romano; la articulación de las piezas de la manica, que se solapaban hacia arriba (a la inversa que la coraza), permitiría una relativa amplia libertad de movimiento al brazo derecho así protegido (Simkins, 1990b: 23-26; Bishop y Coulston, 1993: 87; Coulston, 1998: 5-6; Bishop, 1999: 31-3; Bishop, 2002: 68-71). Esta protección continuó en uso durante el resto del s. II y parte del III, como muestra la escultura mencionada en la nota 22 y datada a fines del s. II o primera mitad del s. III; esta figura puede representar a un legionario -posiblemente de la XIII Gemina- equipado con el típico escudo rectangular semicilíndrico, lorica segmentata (con los hombros protegidos por piezas de squamata) y manica como protección defensiva del brazo de la espada (Coulston, 1995).

27 El listado de asentamientos fuera de Britania (restos en 53 yacimientos), donde se han hallado evidencias de segmentata según M.D. Thomas sería el siguiente:

Alemania: Aislingen, Ausburg-Oberhausen, Baden, Burghöfe, Dangstetten, Eining, Haltern, Häsenbuhl, Heddernheim, Hofheim, Hüfingen, Kalkriese, Magdalensberg, Mainz, Moers-Asberg, Neuss, Oberstimm, Rheingönheim, Rheinzabern, Risstissen, Rottweil, Straubing, Urspring, Wiesbaden, Xanten y Zugmantel.

Austria: Bad Deutsch-Altenberg, Lorch-bi-Enns.

Bélgica: Grobbendonk.

Bulgaria: Svistov.

Chequia: Baden dui Parc, Kaiseraugst, Oberwinterthur, Windisch.

Croacia: Sisak.

España: Herrera de Pisuerga, Pamplona.

Eslovaquia: Iza, Komarno.

Francia: Aulnay-de-Saintonge, Loupain, Estrasburgo, Vaison-la-Romaine.

Holanda: Nijmegen, Valkenburg, Vechten.

Marruecos: Banasa, Thamusida, Volubilis.

Rumanía: Buciumi, Oescus, Porolissum, Sarmizegethusa.

A este listado habría que unir referencias proporcionadas por M.C. Bishop (op. cit., 2002) y que no aparecen en el volumen de M.D. Thomas. Serían las siguientes: Weinberg, León y Carnuntum.

Como vemos, todos estos hallazgos proceden de Europa, a excepción de los atestiguados en el Norte de Marruecos (cf. Thomas, 2003: 2-3). Estos últimos pueden explicarse por la estrecha relación que la provincia de Mauretania Tingitana (que no disponía de unidades legionarias) mantenía con Hispania, por lo que los restos aquí atestiguados podrían haber pertenecido a legionarios de la Tarraconense en misión en estos territorios.

28 Esas evidencias se reducen en muchas ocasiones, como hemos adelantado, a los apliques metálicos de las corazas, enganches o hebillas que, debido a su fragilidad, presentan amplias posibilidades de entrar en el registro arqueológico (ver las categorías de hallazgos $« \mathrm{~A} » \mathrm{a} \ll \mathrm{K} »$ en Thomas, 2003: 6-120); por esta razón hay que tener cuidado con las conclusiones precipitadas respecto a la extensión real de este tipo de protección (Robinson, 1975: 181; Bishop, 1989; Bishop, 1991). 
estaría más extendido entre las tropas legionarias del Oriente Romano que en las propias fronteras europeas, donde el legionario tendría suficiente con la cota de mallas propia del último período de la República ${ }^{29}$.

G. Brizzi, que data la introducción de la lorica segmentata en el segundo cuarto del s. I d.C. ${ }^{30}$, la considera con razón la mejor de todas las que equiparon a los legionarios romanos. De hecho, afirma que fue la adopción de la segmentata y del pilum lastrado la que otorgó la superioridad a las fuerzas romanas en Oriente sobre los contingentes partos, esencialmente compuestos de arqueros a caballo y caballería acorazada (catafractarios) ${ }^{31}$. El autor establece como hipótesis que el pilum lastrado se impuso para resolver un problema táctico preciso, enfrentarse a jinetes acorazados, concretamente catafractarios partos o sármatas. Asímismo, afirma que fue en Oriente donde la lorica segmentata demostró mejor sus cualidades defensivas frente a las flechas de los arqueros a caballo partos ${ }^{32}$. Los restos de este tipo de coraza atestiguados arqueológicamente se concentran, sin embargo, en las fronteras europeas (sobre todo en las antiguas provincias de Britania y ambas Germanias), algo que este autor justifica por el gran desarrollo de los estudios sobre el limes. Además, considera que los descubrimientos realizados hasta ahora permiten suponer una enorme difusión de esta coraza al nivel de todo el Imperio ${ }^{33}$.

G. Brizzi afirma, por otra parte, que la cota de mallas no ofrece protección alguna contra las flechas disparadas por un arco compuesto ${ }^{34}$. Según este autor, este tipo de arco disparaba flechas largas que demandaban una fuerte tensión del brazo, pero que al mismo tiempo debían ser delgadas y ligeras para que su alcance no se viera reducido. Por el contrario, afirma que la lorica segmentata era prácticamente invulnerable a este tipo de flechas si las placas poseían el grosor adecuado, pues al golpear contra una superficie resistente y compacta éstas vibrarían en el momento del impacto, cayendo o rompiéndose. Brizzi sostiene además que una lorica segmentata de $9 \mathrm{~kg}$ de peso presentaría placas de un milímetro de espesor, si bien el espesor real sería el doble al solaparse cada placa con la anterior a lo largo del tronco ${ }^{35}$.

La única representación de segmentata relacionada con los teatros de operaciones orientales son los paneles en relieve del arco de Septimio Severo en Roma, erigido en 206 d.C. No obstante, en estos momentos y sobre todo teniendo en cuenta la influencia de los relieves de las columnas Trajana, Aureliana y Antonina, la lorica segmentata se habría establecido como una convención estilística para representar a las tropas legionarias y diferenciarlas así de las unidades auxiliares ${ }^{36}$.

29 Brizzi, 1981; Brizzi, 2003: 138; Brizzi, 2004: 224-8, 248-9.

30 Ya hemos mencionado, no obstante, que según el registro arqueológico su introducción hay que datarla en la última etapa del reinado de Augusto.

31 Brizzi, 2004: 224.

32 Brizzi, 1981: 198; Brizzi, 2004: 225. Contra M.C. Bishop (2002: 91), que considera poco probable que la segmentata surgiera en Oriente para hacer frente a las flechas de los partos; de hecho, este autor afirma que toda la evolución de este tipo de coraza, desde el modelo Kalkriese al tipo Newstead, se realiza esencialmente con un enemigo en mente: un adversario que ataca mediante potentes golpes descendentes de espada (Bishop, 2002: 98).

33 Cf. nota 29.

34 Contra Bivar, 1972.

35 Compara incluso esta coraza con las armaduras completas del s. XV, que sólo podían ser atravesadas por virotes de ballesta que poseían mayor poder de penetración, sobre todo si el arma contaba con un cuadro de hierro. En cuanto al solapamiento de las placas entre sí, éste se limitaría a ciertas zonas de contacto entre placas; además, las piezas de pectorales y dorsales no se verían reforzadas por solapamiento alguno (véase figura 1).

36 Esto se observa claramente en la Columna Trajana, donde todas las tropas legionarias aparecen invariablemente representadas con la segmentata y los auxiliares con cota de mallas. Los relieves del Tropaeum Traiani de Adamklissi muestran, sin embargo una imagen muy distinta, con legionarios equipados de loricae hamatae y squamatae, pero de un tono general mucho más realista. Por otra parte, la protección propia de la guardia pretoriana en época de Severo era la lorica squamata. 
Existe, no obstante, una noticia de un hallazgo de segmentata en Palestina conectado con la guerra judía de 66-73 d.C. Concretamente, durante la excavación de la fortaleza judía de Gamala, sitiada por Vespasiano en 67 d.C., se halló un fragmento de segmentata muy similar al tipo «Corbridge» ${ }^{37}$. A partir de esta sola evidencia no puede sostenerse, sin embargo, un empleo masivo de este tipo de protección en Oriente, pues esa lorica podía haber llegado allí de muchas formas, entre ellas, por ejemplo, portada por un legionario integrado en alguna vexillatio de tropas procedentes de Europa que hubiera llegado como refuerzo de las unidades que combatían en Palestina esa revuelta judía. Si la extensión de este tipo de protección hubiera sido tan masiva en Oriente como lo fue en Occidente sorprende la ausencia de testimonios de un tipo de coraza que, como hemos visto más arriba, era susceptible de dejar no pocas evidencias arqueológicas en forma de piezas menores ${ }^{38}$.

Se ha pretendido ver una posible evidencia de segmentata en relación con las fronteras orientales en un papiro procedente de Egipto en el que aparece parte de la producción de la fabrica de la II Traiana Fortis acantonada en Nicópolis (junto a Alejandría) ${ }^{39}$. En este papiro, se observa el término «lam(i)nae levisatares», interpretado por R. Marichal como placas de metal para lorica segmentata ${ }^{40}$. Es cierto que la interpretación de «láminas ligeras» que daban los editores puede mantenerse, pero probablemente esas láminas no se referirían a piezas de segmentata, sino a escamas de lorica squamata. En este sentido, Isidoro de Sevilla define a la lorica squamata en los términos siguientes: «squama est lorica ferrea ex laminis ferreis aut aeneis concatenata» (Orig. XVIII, 13, 2); de este modo, esas láminas del papiro de la fabrica de la II Traiana podrían perfectamente hacer referencia a piezas de cotas de escamas. Por otra parte, la datación del papiro a fines del s. III d.C. ${ }^{41}$ tampoco contribuiría a la identificación de esas «lam(i)nae» con componentes de lorica segmentata, reforzando la segunda de las interpretaciones propuestas.

Según Brizzi ${ }^{42}$, un golpe descendente de espada larga, la principal arma junto a la lanza a la que tenían que enfrentarse los legionarios en las fronteras europeas, podía detenerse perfectamente con una cota de mallas, reforzada si acaso con piezas adicionales para los hombros ${ }^{43}$. Sin embargo, la malla absorbía directamente el golpe, trasladando la fuerza del mismo (si bien evitando el corte) al cuerpo del portador, lo que podía provocar graves traumatismos y que los anillos de la coraza pudieran llegar a clavarse en la piel del soldado. Por el contrario, la segmentata, gracias a la estructura de sus hombreras actúa de una forma completamente diferente: en primer lugar detiene mejor el golpe gracias a su rigidez, evitando que se traslade demasiado directamente al legionario, pero también contribuye a desviarlo hacia abajo gracias a la distribución de las láminas de metal en posición descendente ${ }^{44}$. Así, justificar la inoperancia de

37 Cf. Magness y Stiebel, 1995: 8; Peterson, 1996: 16 y 19; este último autor, al igual que Brizzi, también se muestra partidario de extender el empleo masivo de la segmentata a todo el Oriente romano.

${ }^{38} \mathrm{Si}$ bien es cierto que las excavaciones han sido mucho más exhaustivas en las fronteras europeas que en las orientales (como claramente puede observarse en Parker, 2000; Kennedy y Riley, 1990: 122-137), sin embargo, la ausencia de evidencias concretas hacen difícil sustentar ese empleo masivo de la segmentata mencionado por Brizzi o Peterson para este teatro de operaciones.

39 Papiro Berlin inv. 6765 (=ChLA X, 409).

40 Bruckner y Marichal, 1979: 6-7, nº 409 (=PInv 6765, Staatliche Museen Papyrussammlung), línea 10.

41 Según opinión del Prof. Le Bohec (comunicación personal). Los editores del papiro lo fechaban durante el s. II-mediados del s. III.

42 Brizzi, 2004: 249.

${ }^{43}$ Curiosamente las hombreras de las cotas de malla, perfectamente atestiguadas durante el período tardorrepublicano tanto de modelo galo (pequeña capa que cubre los hombros sobre el cuerpo principal de la coraza) como aquellas rectas similares a las de las corazas de lino de tipo griego más influidas por modelos helenísticos desaparecen durante el s. II d.C. (cf. Menéndez Argüín, 2004: 210).

44 Brizzi (2004: 249) afirma que este problema se podría haber solucionado aplicando a la cota de mallas hombreras similares a las de la lorica segmentata, pero este híbrido no se ha atestiguado hasta ahora en el registro arqueológico y sólo un investigador ha pretendido verlo en una fuente de carácter iconográfico (relieve de jine- 
la segmentata en las fronteras europeas nos parece, como poco, arriesgado. Además, hay que recordar que este tipo de coraza surge en combinación con nuevos tipos de casco, que precisamente contribuyen a reforzar la protección de nuca y hombros contra golpes descendentes, actuando como elementos deflectores hacia la coraza que, como hemos visto, contribuía definitivamente a que el golpe se perdiera causando al soldado el menor daño posible.

En cuanto a la adopción de esta coraza por parte de las legiones orientales para hacer frente a las flechas disparadas por los partos desde un arco compuesto, según Brizzi caracterizadas por ser largas y delgadas, lo primero que puede decirse es que tanto el tamaño de estas últimas, como su peso y la forma de la punta dependían del tamaño del arco, el uso a las que se las iba a destinar y el grado de protección del objetivo, por lo que no nos parece adecuada una visión tan reduccionista ${ }^{45}$. Por otra parte, el alcance, según estimaciones modernas oscilaba entre 64 y $600 \mathrm{~m}^{46}$. En una publicación anterior he discutido este aspecto más detenidamente ${ }^{47}$, baste decir aquí que hay que distinguir claramente entre alcance máximo (distancia total a la que el esfuerzo físico de la propulsión puede mandar la flecha, aunque no llegaría con la fuerza suficiente para producir daños sobre el objetivo) y alcance eficaz (distancia máxima a la que puede ser arrojada la flecha y producir efectos sobre el objetivo); este último, no obstante, no puede calcularse de una forma que pudiera calificarse de definitiva, pues dependería tanto del tipo de flecha utilizada como del grado de protección del objetivo (mayor protección, menor distancia eficaz del arco). Como referencia puede mencionarse que un buen arco compuesto es capaz de atravesar una coraza a cien metros de distancia ${ }^{48}$; ésta podía ampliarse, por supuesto, en caso de objetivos no acorazados o livianamente protegidos. Por tanto, hablar de invulnerabilidad de la lorica segmentata ante las flechas partas, sin negar por supuesto sus excelentes cualidades defensivas, nos parece excesivo. Los partos podían haber modificado el tipo de punta empleada, el tipo de astil de la flecha, la distancia a la que atacaban el objetivo, etc. ${ }^{49}$ para hacer frente a este avance defensivo del enemigo ${ }^{50}$.

tes de Arlon, en la frontera entre Bélgica y Luxemburgo, véase infra) (Simkins, 1988: 122). Una obra apropiada para haber representado este nuevo tipo de coraza en relación con la infantería, si realmente hubiera existido, podía haber sido el monumento de Adamklissi, que en cambio sí que representa las manicae lamminatae adoptadas para combatir a los dacios. Aun así, la fusión entre distintos tipos de defensas corporales es algo bastante lógico: la escultura de Alba Iulia (véase nota 22) muestra la fusión entre lorica squamata y segmentata, si bien en un sentido inverso al propuesto por Brizzi, pues son los hombros los que aparecen protegidos por piezas de escamas, en lugar de mantener las hombreras propias de la segmentata, en teoría mejor adaptadas para la defensa de esta parte del tronco. Por contra, en un relieve de Arlon que muestra a varios jinetes romanos al ataque, M. Simkins ha interpretado la coraza que portan como una cota de mallas reforzada en los hombros por piezas de lorica segmentata (esta vez sí en la forma propuesta por el investigador italiano); el único inconveniente, si esta reconstrucción se acepta, es que se trata de tropas a caballo, no de infantería legionaria. M.C. Bishop, desechando la interpretación anterior, opina que se trataría de una mala interpretación por parte del escultor de las hombreras propias de la cota de malla. La escasa calidad del relieve no contribuye tampoco a facilitar las cosas (cf. Bishop, 2002: 72-73).

45 Cf. Coulston, 1985: 220-366. Si bien este autor se refiere a arcos y flechas empleadas por el ejército romano, suponemos que estas premisas serían, como es lógico, conocidas y aplicadas por los arqueros partos.

46 McLeod, 1965; McLeod, 1972.

47 Menéndez Argüín, 2000a: 157-158.

48 Cf. Keegan, 1995: 205-206.

49 La prueba de que la lorica segmentata no era invulnerable a las flechas la tenemos en un hallazgo de Catalka (Bulgaria), en el que se ha conservado una punta de flecha incrustada en el metal de un fragmento de este tipo de coraza (cf. Bujukliev, 1986: n. 96 del catálogo; Bishop, 2002: 84).

50 Otro problema diferente es que evitaran todo lo posible el contacto con la infantería de línea, pues el sistema militar parto, basado en la caballería pesada y ligera, no contemplaba un auténtico enfrentamiento con la línea de infantería pesada del enemigo, sino su progresivo debilitamiento que culminaba con una carga de caballería que terminaba de disolverla. Más que en el equipamiento de los legionarios, la supremacía de los romanos en Oriente a lo largo de todo el Alto Imperio habría que buscarla en un desarrollo magistral de la táctica de armas combinadas, con fuertes unidades ligeras de caballería e infantería (en un intento de combatir en pie de igualdad contra el parto), unidas a un fuerte núcleo de infantería pesada que les permitía retirarse y reorganizarse (algo de lo que carecía el enemigo) en caso de contraataques en fuerza. 
Por otra parte, como claramente se observa en la descripción de la formación en testudo de la batalla de Carras, hay que tener en cuenta que la infantería pesada en pocas ocasiones presentaría el tronco al adversario, al cubrirse lo más posible con el propio escudo y con el del compañero de la derecha; sólo parte de los hombros, parte inferior de las piernas y la parte superior del rostro serían susceptibles de sufrir los impactos de las nubes de flechas arrojadas por el enemigo; estas superficies en peligro se reducían aún más con el empleo de la testudo con carácter estático. Sólo tras el colapso moral de las tropas pudieron los partos conseguir la victoria en 53 a.C., al desagregarse y acabar exponiéndose unos a otros a los disparos enemigos. En este sentido, lo que sí pudo haberse mantenido en uso en Oriente incluso más allá de su abandono por parte de las legiones situadas en las fronteras europeas es el scutum rectangular semicilíndrico, como muestra el ejemplar de mediados del s. III hallado en Dura Europos, gracias a la superior protección que proporcionaba contra las flechas de los arqueros partos y persas.

El profesor Brizzi aduce también para apoyar su teoría el pasaje de Frontón en el que los legionarios de Trajano después de haberse enfrentado a las espadas curvas de los dacios despreciaban las flechas de los partos ${ }^{51}$. Pero el problema es saber con qué tipo de coraza se enfrentaron estos soldados a los dacios; recordemos que en el monumento de Adamklissi no aparece representada ni una sola lorica segmentata, sino soldados con cota de mallas o escamas haciendo frente a las falces dacias. Por ello esta apreciación no nos parece concluyente. Más difícil de aceptar es la conclusión extraída de un pasaje de Vegecio (Epit. II, 15) en el que Brizzi afirma que la coraza estaba pensada para proteger de las flechas, y que esa coraza debía ser la segmentata. En primer lugar, el pasaje de Vegecio está describiendo las distintas armas que equipaban a la infantería pesada y, cuando llega al pilum, simplemente describe su actuación, afirmando que bien arrojado era capaz de atravesar tanto a los infantes pesados (scutatos pedites) como a los jinetes acorazados (loricatos equites); por otra parte, podemos sobreentender que Vegecio hace referencia al enemigo exterior, entendiendo por scutatos pedites los pueblos germanos (entre los que las protecciones corporales no abundaban precisamente) y por loricatos equites a la caballería pesada de tipo oriental (catafractarios, clibanarios), lo que da a entender perfectamente la gran utilidad de este tipo de pilum. Por otra parte, Brizzi hace referencia sin citarlo al pasaje I, 20, en el que se habla de la falta de protección de la infantería desde la época de Graciano para hacer frente a los ataques de los arqueros godos. Pero de aquí no se infiere en absoluto una coraza especialmente adaptada para proteger contra las flechas, simplemente Vegecio, en un tono tremendista, hace referencia al abandono total de las protecciones corporales (incluido el casco) por parte de la infantería de los últimos años del s. IV ${ }^{52}$.

Una cuestión que cabría plantearse es por qué, si las cualidades de la segmentata eran tan extraordinarias, nunca se intentó una adaptación de la misma a la caballería pesada que, como los clibanarii o contarii que combatían en las fronteras orientales, luchaba sin escudo y en la que toda protección adicional hubiera sido muy bien recibida ${ }^{53}$. En ningún momento se nos describe un jinete pesado equipado con algo parecido ni remotamente a la seg-

51 Frontón, Principia Historiae 9.

52 Sobre el supuesto abandono de las protecciones corporales durante los ss. III-IV remitimos a: Coulston, 1990; Menéndez Argüín, 2000a: 111-115; Beltrán Fortes y Menéndez Argüín, 1999; Menéndez Argüín, 2000b; Beltrán Fortes y Menéndez Argüín, 2001.

53 Además, tanto la caballería romana en Oriente como los arqueros reclutados en estas zonas estaban equipados con cotas de malla o escamas, a pesar de ser los que más expuestos estarían a las flechas de los arqueros partos (pues eran los que tenían más posibilidades de entrar en contacto con el enemigo); sin embargo, no se realiza intento alguno de aplicarles la segmentata, algo que, si la cota de mallas hubiera sido tan deplorable defensivamente frente a las flechas como afirma Brizzi podía haber reducido ampliamente el número de bajas entre estos cuerpos de tropas. 
mentata, sino que el material del que solía estar constituida su armadura eran anillos, escamas o pequeñas láminas de metal. Podrían aducirse problemas de movilidad, pero problemas similares o peores provocaban las armaduras completas del s. XV y no por ello dejaron de emplearse y reforzarse. Además, los catafractarios y clibanarios equipados en malla también carecían de la movilidad adecuada para acometer maniobras que no fueran la carga directa o para sobrevivir desmontados. La razón habría que buscarla en que la zona en la que se combatía, el Próximo Oriente, no favorecía la adaptación de corazas más o menos rígidas de placas de metal (ver infra), favoreciéndose sobre todo la malla como el mejor compromiso entre protección y portabilidad. Aun así, los jinetes fuertemente acorazados recibieron el nombre de clibanarios probablemente en referencia al sofocante calor que sufrían bajo las protecciones que endosaban ${ }^{54}$; si encima la coraza hubiera estado formada por placas de metal rígido, la sensación de calor hubiera sido completamente insoportable durante los períodos de campaña ${ }^{55}$.

Otro problema añadido es el de la corrosión ${ }^{56}$, que en Oriente habría afectado a este tipo de corazas de placas de una forma mucho mayor que a la malla, reduciendo la vida útil de las piezas y obligando a un importante gasto en mantenimiento o reposición de equipo. En efecto, la acidez de la transpiración deteriora rápidamente las piezas más frágiles, requiriendo el envío de la coraza a la armería para su reparación, como se ha podido comprobar en numerosas reconstrucciones modernas ${ }^{57}$. Si este problema es apreciado por grupos de reconstrucción modernos en zonas relativamente frías del Occidente de Europa (Gran Bretaña, Holanda, Norte de Francia, Alemania), no es complicado imaginar que el empleo de la segmentata en Oriente habría sido una auténtica pesadilla logística para la maquinaria militar romana.

Además, la lorica segmentata era una coraza diseñada esencialmente para el combate cuerpo a cuerpo ${ }^{58}$. Pero precisamente este tipo de combate era extremadamente raro para la infantería pesada en las operaciones en Oriente; por todo ello, podemos preguntarnos si merecía la pena hacer sufrir a los legionarios en este frente todos los inconvenientes que planteaba la coraza de placas en un tipo de guerra que no solía demandar de ellos el choque directo contra el enemigo ${ }^{59}$.

Finalmente puede traerse a colación el pasaje de Julio Africano en el que describe (bien es cierto que en un tono bastante crítico) el equipamiento de la infantería propia de las legiones orientales durante el reinado de Severo Alejandro $(222-235)^{60}$. Este autor nos informa que el infante romano iba equipado con cota de malla, una greba, yelmo, escudo de un solo asidero, spatha y varias jabalinas. Julio Africano critica el escudo (inadecuado para la táctica de falange), el casco (que restringía demasiado la visión y los movimientos de la cabeza del soldado), las armas de asta (incapaces de resistir una carga de caballería)... pero no critica la cota de mallas, lo que haría suponer que era una protección suficiente y adecuada para los solda-

54 El término provendría de la palabra latina «clibanus», horno de campaña.

55 El problema del calor no debe ser minusvalorado, sobre todo en relación con tropas no acostumbradas al mismo, como muestra claramente un pasaje de Zósimo: «Mientras Probo dilataba la guerra (...) sobrevino el calor estival en Tarso, a causa del cual las tropas de Floriano, no habituadas por proceder de Europa el grueso de su ejército, cayeron gravemente enfermas» $(64,2)$. Por otra parte, tanto en Oriente como en África la malla siguió en uso siglos después de haberse creado en Europa las armaduras rígidas; esa continuidad no puede ser sino indicio de su eficacia y adaptación a las condiciones climáticas de estos territorios.

56 Cf. Bishop, 2002: 80-1.

57 Cf. Gilbert, 2004: 48.

58 El hecho de que sólo fuera portada por la infantería pesada legionaria es buena muestra de esta afirmación.

59 A esto habría que unir el mayor volumen de gasto para el Estado derivado del mantenimiento y reposición de este tipo de corazas, que incrementaría desproporcionada e innecesariamente las necesidades logísticas de estas tropas en relación con el enemigo al que tenían que hacer frente.

60 Cest. fr. 1.1.50-56. 
dos que se servían de ella en esta zona fronteriza del Imperio. Asimismo, Casio Dion ${ }^{61}$ pone en boca de un oficial de Septimio Severo durante el asedio de Hatra que con 550 hombres procedentes de las fronteras europeas (es decir, una cohorte) habría tomado sin problemas la fortaleza. En esta noticia, además de observarse el secular y tópico criticismo contra la operatividad de las tropas de las guarniciones legionarias de Oriente, puede que quizás se haga también referencia a las mejores defensas corporales empleadas por los soldados estacionados a lo largo del Rin y el Danubio, a los que la lorica segmentata protegía mejor para enfrentarse a operaciones de asedio y asalto. El hecho de que las tropas orientales, si se acepta esta interpretación del pasaje, carecieran de esas protecciones sería un handicap insuperable que se saldaría con el doble fracaso de Severo ante esta fortaleza.

Para concluir, queremos señalar que no negamos la posibilidad de que este tipo de coraza se empleara en los teatros de operaciones del Oriente Romano, simplemente queremos resaltar su inadecuación al medio y la imposibilidad, en el estado actual de nuestros conocimientos, de ver un empleo similar al que habría tenido la lorica segmentata en las unidades legionarias de las fronteras europeas.

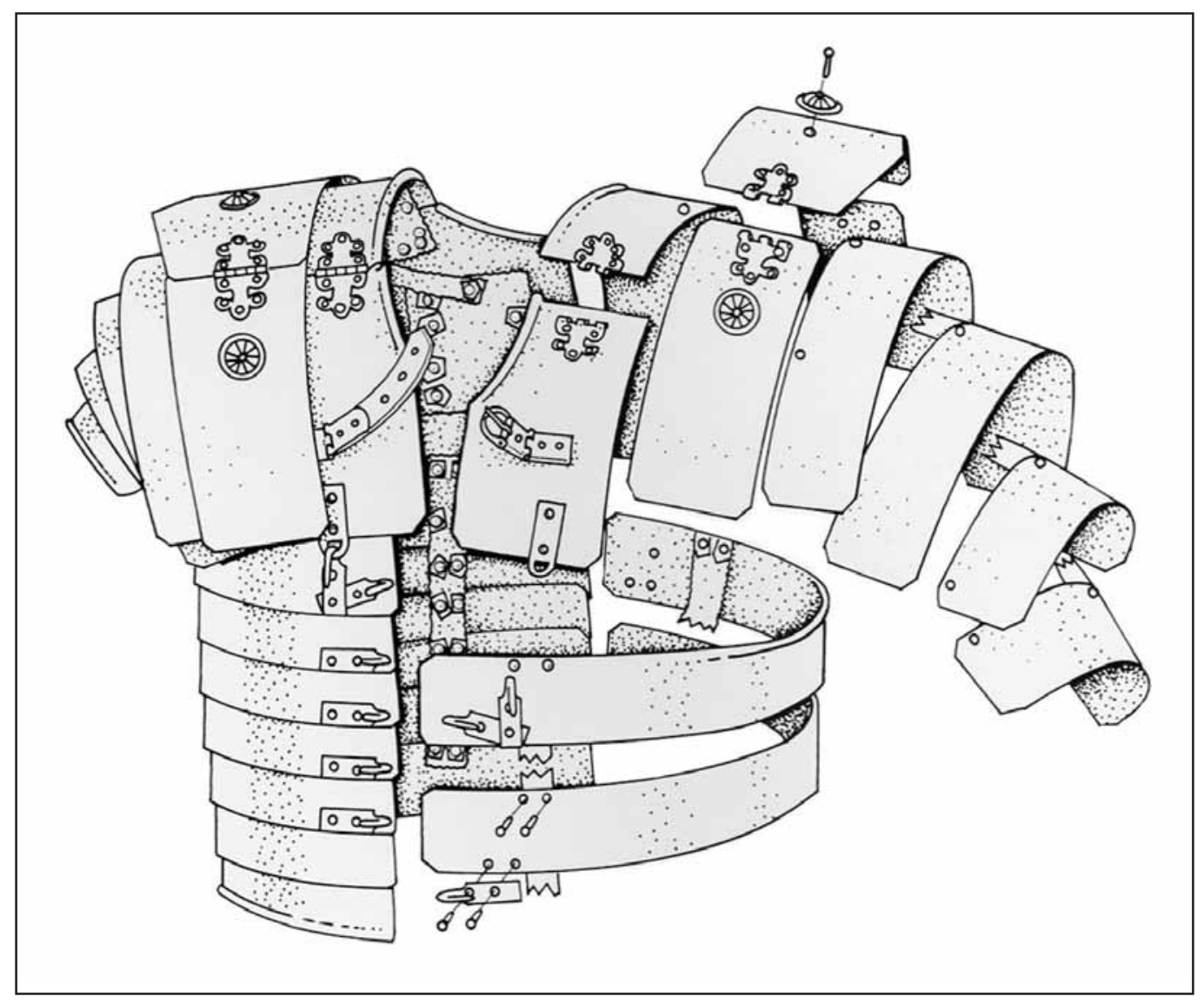

Fig. 1. Lorica segmentata tipo «Corbridge».

61 D.C. 75.12 .5 . 


\section{BIBLIOGRAFÍA}

Beltrán Fortes, J. y Menéndez Argüín, A. Raúl (1999): «New Evidence on the use of armour by roman soldiers in the fourth century AD», JRMES 10: 17-23.

Beltrán Fortes, J. y MenÉndez Argǘn, A. Raúl (2001): «Sobre el armamento defensivo de los soldados romanos en el siglo IV d.C. A propósito de un relieve de Córdoba», Habis 32: 505-520.

BiAnCHI, L. (1988): «Adamclisi: il programa storico e iconografico del trofeo di Traiano», RSA 2: 427473.

Bishop, M. C. (1989): «O Fortuna: A sideways look at the Archaeological Record and Roman Military Equipment», en VAn Driel-Murray, C. (ed.), Roman Military Equipment: the Sources of Evidence, Oxford: 1-11.

Bishop, M. C. (1991): «Soldiers and Military Equipment in the Towns of Roman Britain», en MAXFIELD, V. A. y Dobson, B. (eds.), Roman Frontier Studies 1989, Exeter: 21-27.

Bishop, M. C. (1999): «The Newstead 'lorica segmentata'», JRMES 10: 27-43.

Bishop, M. C. (2002): Lorica segmentata. Volume I: A Handbook of Articulated Roman Plate Armour, JRMES Monograph N. ${ }^{\circ}$ 1, Oxford.

Bishop, M. C. y Coulston, J. C. N. (1993): Roman Military Equipment from the Punic Wars to the Fall of Rome, Londres.

Bivar, A. D. H. (1972): «Cavalry Tactics and Equipment on the Euphrates», Dumbarton Oak Papers 26: 273-291.

Brilliant, R. (1967): The Arch of Septimius Severus in the Roman Forum, MAAR 29, Roma.

BRIZZI, G. (1981): «L'armamento legionario dall'età giulio-claudia e le guerre partiche», Critica Storica 18, 2: 177-201.

BrizZi, G. (2003): «L'età di Adriano: armamenti e tattiche», en LE BoHEC, Y. (ed.), Les Discours d'Hadrien à l'armée d'Afrique, Paris.

Brizzi, G. (2004): Le guerrier de l'Antiquité Classique. De l'hoplite au légionnaire, Paris.

Bruckner, A. y MARIChal, R. (1979): Chartae latinae antiquiores X, Berlín.

Bujukliev, H. (1986): La Nécropole Tumulaire Thrace près de Catalka. Region de Stara Zagora, Sofia.

Caruana, I. (1993): «A Third Century Lorica Segmentata back-plate from Carlisle», ARMA 5.2 (Dic. 1993): $15-18$.

Cichorius, C. (1896-1900): Die Reliefs der Traianssäule, Berlin, 7 vols.

CoArelli, F. (1980): Roma (Guide Archeologiche Laterza), Roma.

Coulston, J. C. N. (1985): «Roman Archery Equipment», en Bishop, M. C., The Production and Distribution of Roman Military Equipment: Proceedings of the Second Roman Military Equipment Seminar (BAR 275), Oxford: 220-366.

Coulston, J. C. N. (1990): «Later Roman Armour, 3rd-6th Centuries AD», JRMES 1: 139-160.

Coulston, J. C. N. (1995), «The Sculpture of an Armoured Figure at Alba Iulia, Romania», ARMA 7: 13-17.

Coulston, J.C.N. (1998): «Gladiators and Soldiers: Personnel and Equipment in ludus and castra», JRMES 9: 1-17.

Cowan, R. (2004): Le légionnaire romain de 58 av.J.-C. à 69 apr. J.-C., Paris.

Feugère, M. (1993): Les Armes des Romains. De la République à l'Antiquité Tardive, París.

FIngerlin, G. (1986): Dangstetten I. Katalog der Funde (Fundstellen 1 bis 603), Stuttgart.

Fingerlin, G. (1998): Dangstetten II. Katalog der Funde (Fundstellen 604 bis 1358), Stuttgart.

Florescu, F.B. (1965): Das Siegesdenkmal von Adamklissi: Tropaeum Traiani, Bucarest.

Florescu, R. (1998): «Il Tropaeum Traiani di Adamklissi», en G. A. PoPeSCU (dir.), Traiano ai confini dell'Impero, Milan: 148-155.

GILBERT, François (2004): Le soldat romain à la fin de la République et sous le Haut-Empire, Paris.

HARMAND, J. (1987): «L'Armement défensif romain de métal dans le nord-ouest de l'Empire, de la conquête au $\mathrm{V}^{\mathrm{e}}$ siècle», en Les Mines et la Metallurgie en Gaule et dans les Provinces Voisines, Caesarodunum 22, Actes du Colloque, Paris: 189-203.

KeEgAn, J. (1995): Historia de la Guerra, Barcelona.

KenNedy D., y Riley, D. (1990): Rome's Desert Frontier from the Air, Londres. 
LeAnder Touati, A.M. (1987): The Great Traianic Frieze. The Study of a Monument and of the Mechanisms of Message Transmission in Roman Art, Estocolmo.

Magness, J. y Stiebel, G.D. (1995): «Roman Military Equiment from Gamla», ARMA 7: 8.

McLeod, W. (1965): «The Range of the Ancient Bow», Phoenix 19: 1-14.

MCLeod, W. (1972): «The Range of the Ancient Bow: Addenda», Phoenix 26: 78-81.

MenÉndez Argüín, A. Raúl (2000a): Las legiones del s. III d.C. en el campo de batalla, Écija (Sevilla).

Menéndez Argüín, A. Raúl (2000b): «Evolución del armamento del legionario romano durante el s. III d.C. y su reflejo en las tácticas», Habis 31: 327-344.

Menéndez Argüín, A. Raúl (2004): Las legiones romanas de Germania (ss. II-III d.C.): Aspectos logísticos, Écija (Sevilla).

PARKER, S. T. (2000): «Roman Legionary Fortresses in the East», en R.J. Brewer (ed.), Roman Fortresses and their Legions, Londres: 121-135.

Petersen, E., Von Domaszewski, A. y Calderini, G. (1896): Die Marcus-Saule auf der Piazza Colonna in Rom, Múnich.

Peterson, D. (1996): The Roman Legions Recreated in colour Photographs, London.

Robinson, H. Russell (1974): «Roman Body Armour in the First Century A.D.», en BiRLEY, E., DobSON, B. y JARretT, M. (eds.), Roman Frontier Studies 1969. Eight International Congress of Limesforschung, Cardiff: 5-12.

Robinson, H. Russell (1975): The Armour of Imperial Rome, Londres.

Roth-Rubi, K., «La sigillée de Dangstetten: question de chronologie», en Acta Rei Cretariae Romanae Fautorum, Acta 37 (Lyon 2000), Abingdon 2001: 211-215.

SCHLÜTER, W. et alii (1992): «Archäologische Zeugnisse zur Varusschlacht? Die Untersuchungen in der Kalkriese-Niewedder Senke bei Osnabrück», Germania 70: 307-402.

SCHLÜTER, W. (1999): «The Battle of the Teutoburg Forest: Archaeological Research at Kalkriese near Osnabrück», en WiLson y CREIGHTON (eds.), Roman Germany. Studies in Cultural Interaction, Portsmouth (Rhode Island): 125-159.

Simkins, M. (1986): The Roman Army from Caesar to Trajan, London.

SimKINS , M. (1988): Warriors of Rome, Londres.

SIMKINS, M. (1990a): «Lorica Segmentata?», ARMA 2.1 (Jun. 1990): 11.

Simkins, M. (1990b), «The Manica Lamminata», ARMA 2.2 (dic. 1990): 23-26.

TARPIN, M. (2001): Roma Fortunata, Paris.

Thomas, M. D. (2003): Lorica segmentata. Volume II: A Catalogue of Finds, JRMES Monograph N. ${ }^{\circ}$ 2, Oxford.

TrIER, B. (ed.) (1989): 2000 Jahre Römer in Westfalen, Mainz.

TURCAN, R. (1995): L'art romain, Paris.

WILKINS, A. (2000): «Scorpio and cheiroballistra», JRMES 11: 77-101.

Wilson, R. J. A. y Creighton, J. D. (1999): «Recent Research on Roman Germany», en Id. (eds.), Roman Germany. Studies in Cultural Interaction, Portsmouth (Rhode Island): 9-34.

ZwIKKER, W. (1941): Studien zur Markussäule, Archaeologisch-Historische Bijdragen Deel VIII, Amsterdam.

Recibido: 30/09/2005

Aceptado: 05/10/2005 